Pneumologe 2012 · 9:319-320

DOI 10.1007/s10405-011-0519-1

Online publiziert: 29. August 2012

๑) Springer-Verlag 2012

\author{
N. Konietzko \\ Essen
}

\title{
Die Lunge im Alter
}

sich von all dem nicht haben entmutigen lassen, die relevanten Daten zusammengetragen und mit großer Sorgfalt in ihre Beiträge eingearbeitet haben.

Im ersten Beitrag zur „Epidemiologie der Lungenkrankheiten" analysieren $V e$ rena Knipel, Wolfram Windisch und Abdel Hakim Bayarassou zunächst die Folgen des demographischen Wandels in Deutschland im Allgemeinen und für unser Gesundheitswesen im Speziellen. Sodann wagen sie eine Prognose der weiteren Entwicklung bis in die Jahrhundertmitte hinein. Bei abnehmender Gesamtbevölkerung werden dann $40 \%$ aller Deutschen älter als 60 Jahre sein. Erkrankungen der Atemorgane werden in absoluten Zahlen bis 2050 weiter zunehmen, speziell die pneumologischen Volkskrankheiten: der Lungenkrebs um $26 \%$, die COPD um 23\% und die ambulant erworbene Pneumonie um 140\%. Die Gesundheitskosten, die zukünftig für Erkrankungen der Lunge von der Solidargemeinschaft aufgebracht werden müssen, lassen sich nur grob schätzen, werden aber überproportional wachsen, da zumindest die direkten Gesundheitskosten im Alter exponentiell zunehmen [1].

Im zweiten Beitrag zur „Funktion der Lunge im Alter" erklärt Helmut Teschler klar, warum das viel zitierte „Altersemphysem“, die „welke Alterslunge“ der Pathologen, keine Krankheit ist, auch wenn sich morphologisch ein Verlust kollagener Fasern als Ausdruck des über ein langes Leben kumulierten oxydativen Stresses nachweisen lässt. Entsprechend vermindert sich die Lungenfunktion mit den Jahren. Allerdings altert die Lunge, verglichen mit anderen Organen, relativ langsam. Extrapoliert man den $\mathrm{FEV}_{1}$-Verlust $(\triangle \mathrm{FEV} 1)$ von $34 \mathrm{ml}$ pro Jahr beim Mann, den Helmut Teschler in seinem Beitrag zitiert [2], und beginnt dabei mit dem
30. Lebensjahr, so käme man auf das biblische Lungenalter von 180 Jahren, wenn man einen Atemstoß von Null als definitiven Endpunkt setzt. Natürlich ist eine solche lineare Extrapolation nicht zulässig, doch sei der Schluss erlaubt, dass eine gesunde Lunge für die statistische Lebenserwartung nicht limitierend ist. $\mathrm{Zu}$ den weniger erfreulichen Erkenntnissen gehört freilich die Tatsache, dass die Effektivität des Immunsystems im Alter nachlässt. Sowohl die unspezifischen, angeborenen wie auch die erworbenen Immunmechanismen funktionieren im Alter nicht mehr so effektiv [3]. Die Folgen zeigen sich in einer erhöhten Anfälligkeit gegenüber exogenen Noxen, insbesondere gegen pathogene Mikroorganismen und Staubpartikeln.

\section{\) Medikamentenverbrauch und Nebenwirkungen nehmen mit dem Alter zu}

Patienten über 65 Jahre schlucken im Durchschnitt 5-mal so viele Tabletten wie jüngere Menschen. Das ist an und für sich nicht verwunderlich, da im gleichen Zeitraum auch die Multimorbidität ansteigt, jedoch sind ältere Menschen anfälliger für unerwünschte Nebenwirkungen. Zudem haben viele Senioren Probleme mit der regelmäßigen Einnahme und der richtigen Dosierung ihrer Medikamente. In ihrem Beitrag zur „Pharmakotherapie im Alter" zeigen Alexandra Wald und Hubertus Wirtz die Problematik der Polypharmazie und ihrer Auswirkungen auf die Toxizität. Sie kann im Alter dramatische Ausmaße annehmen. Mit dem Trick der lokalen Applikation von Medikamenten in unserem Fachgebiet Standardtherapie in Form der Inhalation von Steroiden und
Umso dankbarer bin ich den Autoren des vorliegenden Themenhefts, dass sie 
Betamimetika - wird zwar die Gesamtdosis für den Organismus reduziert und damit auch die Toxizität, aber die Vielzahl der Inhalationsgeräte und -techniken überfordert alte Menschen oft.

Erst der konsequenten Impfung gegen die „großen“ Infektionskrankheiten im Kindesalter, die Ende des 18. Jahrhunderts mit Edward Jenners Pockenvakzine in die moderne Medizin Einzug hielt, ist es zu verdanken, dass heute immer mehr Menschen ein respektables Alter erreichen und dabei auch noch fit bleiben. Aber auch im Alter können Impfungen trotz nachlassender Impfreaktion wirksam und ihr Einsatz sinnvoll sein, das zeigen Anja Kwetkar, Stefan Hagel und Mathias Pletz in ihrem Beitrag „Impfungen im Alter". Sie weisen auch Wege, wie die Immunseneszenz, die nachlassende Immunreaktion im Alter, überspielt werden kann, etwa durch intradermale Injektion des Impfstoffs statt der üblichen subkutanen oder durch Modulation der Vakzine [3]. Wie segensreich eine konsequente Durchimpfung in der Kindheit sich für alte Menschen auswirken kann, zeigt sich eindrücklich am Beispiel der Pneumokokken-Impfung im Kindesalter, die seit gut 20 Jahren im STIKO-Impfprogramm enthalten ist. Durch sie wird die Pneumokokken-Besiedlung des kindlichen Atemtrakts, dem wichtigsten Reservoir dieser Keime, reduziert. Dadurch geht letztlich auch die Zahl der Pneumokokken-Pneumonien im Alter zurück.

Altern ist keine Krankheit, Altern ist eine Erscheinungsform des Lebens. Jedoch nehmen chronische Krankheiten im Alter zu, treten häufig gemeinsam auf und erhöhen das Risiko eines vorzeitigen Todeseintritts. Sind die Grenzen der Therapie erreicht, drängen sich Fragen der ärztlichen Ethik mehr und mehr in den Vordergrund [4]. Sie stehen auch im Zentrum meines Beitrags über „Die Grenzen der Therapie“. Neben Fragen der ärztlichen Ethik wird aber auch juristischen Aspekten zu Grenzthemen am Lebensende Raum gegeben. Anhand konkreter Beispiele aus der ärztlichen Praxis wird das Dilemma zwischen Autonomie des Patienten und der Fürsorgepflicht des Arztes, vor das der Arzt immer wieder gestellt wird, anschaulich gemacht und nach Lösungen gesucht. Zwangsläufig fließen in den vorliegenden Beitrag auch meine eigenen Lebens- und Berufserfahrungen mit ein. Er kann somit nur meine persönliche, der Kritik offene Sicht auf ethische Fragen des ärztlichen Alltags aufzeigen und keinen Anspruch auf Allgemeingültigkeit erheben.

Den Autoren danke ich für die sorgfältige Darstellung der Themen dieses Schwerpunkthefts und den Damen des Springer-Verlags für die bewährte $\mathrm{Zu}$ arbeit. Den Lesern wünsche ich eine anregende Lektüre.

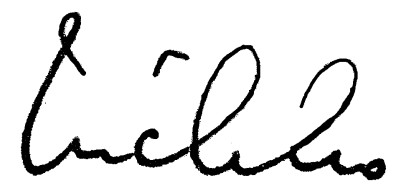

Nikolaus Konietzko

\section{Korrespondenzadresse}

Prof. Dr. N. Konietzko

Spillheide 78, 45239 Essen

nikolaus.konietzko@t-online.de

\section{Literatur}

1. Beske F et al. (2009) Morbiditätsprognose 2050. Ausgewählte Krankheiten für Deutschland, Brandenburg und Schleswig-Holstein. Schriftenreihe/ Fritz Beske Institut für Gesundheit-System-Forschung Kiel; Bd. 114

2. Frette C, Barrett-Connor E, Clausen JL (1996) Effect of active and passive smoking on ventilatory function in elderly men and women. Am J Epidemiol 143:757-765

3. Meyer KC (2010) The role of immunity and inflammation in lung senescence and susceptibility to infection in the elderly. Semin Respir Crit Care Med 31:561-574

4. Maio G (2012) Mittelpunkt Mensch. Ethik in der Medizin. Schattauer-Verlag, Stuttgart

\section{Online-Zugriff von SDGP- Website auf Der Pneumologe}

Abonnenten der Zeitschrift Der Pneumologe haben kostenfreien Zugriff auf das SpringerLink-Online-Archiv, welches neben der aktuellen Ausgabe der Zeitschrift auch alle bisher erschienenen Beiträge enthält. Sie erreichen das Online-Archiv unter der Adresse www.DerPneumologe.de über den Button "Read online".

Für alle Mitglieder der Süddeutschen Gesellschaft für Pneumologie ist auf der Homepage der SDGP (www.sdgp.de) im internen Bereich ein direkter Zugang eingerichtet. Dieser ermöglicht nach Anmeldung als Mitglied der SDGP den Zugriff auf das Volltext-Archiv von Der Pneumologe ohne zusätzliches Log-in.

Viel Freude bei der Lektüre wünscht Ihnen Ihre Redaktion Der Pneumologe 